\title{
Initial Management and Outcomes After Superficial Thrombophlebitis: The Cardiovascular Research Network Venous Thromboembolism Study
}

\author{
Bethany Samuelson, MD¹, Alan S. Go, MD²,3, Sue Hee Sung, $\mathrm{MPH}^{2}$, Dongjie Fan, $\mathrm{MSPH}^{2}$, Margaret C. Fang, MD, MPH${ }^{4 *}$
}

\begin{abstract}
${ }^{1}$ Division of Hematology-Oncology, University of Washington, Fred Hutchinson Cancer Research Center, Seattle, Washington; ${ }^{2}$ Division of Research, Kaiser Permanente Northern California, Oakland, California; ${ }^{3}$ Departments of Epidemiology, Biostatistics, and Medicine, University of California, San Francisco, San Francisco, California, and Department of Health Research and Policy, Stanford University School of Medicine, Palo Alto, California; ${ }^{4}$ Division of Hospital Medicine, University of California, San Francisco, San Francisco, California.
\end{abstract}

\begin{abstract}
Although superficial thrombophlebitis (SVTE) is generally considered a benign, self-limited disease, accumulating evidence suggests that it often leads to more serious forms of venous thromboembolism. We reviewed the medical charts of 329 subjects with SVTE from the Cardiovascular Research Network Venous Thromboembolism cohort study to collect information on the acute treatment of SVTE and subsequent diagnosis of deep venous thrombosis within 1 year. All participants received care within Kaiser Permanente Northern California, a large, integrated healthcare delivery system. Fourteen $(4.3 \%)$ subjects with SVTE
\end{abstract}

received anticoagulants, $148(45.0 \%)$ were recommended antiplatelet agents or nonsteroidal anti-inflammatory drugs, and in 167 (50.8\%) there was no documented antithrombotic therapy. In the year after SVTE diagnosis, 19 (5.8\%) patients had a subsequent diagnosis of a deep venous thrombosis or pulmonary embolism. In conclusion, clinically significant venous thrombosis within a year after SVTE was uncommon in our study despite infrequent use of antithrombotic therapy. Journal of Hospital Medicine 2016;11:432434. (C) 2016 Society of Hospital Medicine
Superficial thrombophlebitis (SVTE), inflammation of superficial veins associated with thrombosis, is a painful condition, and $\sim 3 \%$ to $11 \%$ of the population will develop SVTE during their lifetime. Although generally considered a benign, self-limited disease, it can cause considerable discomfort, impact mobility, and lead to further complications. Recent and accumulating evidence suggests that it is often associated with more serious forms of venous thromboembolism (VTE), including deep vein thrombosis (DVT) and pulmonary embolism (PE), ${ }^{1}$ and SVTE is a strong risk factor for subsequent DVT or PE. ${ }^{2,3}$

There is no clear consensus on the optimal treatment of SVTE. Although antithrombotic medications such as fondaparinux may be more effective than placebo in reducing the risk of subsequent $\mathrm{DVT},{ }^{4}$ the evidence is generally of low grade, and the costs and inconveniences of anticoagulant therapy are not inconsequential. ${ }^{1,5,6}$ Surveys suggest that physician opinions on the appropriate management of SVTE vary significantly, and management includes nonsteroidal anti-inflammatory drugs (NSAIDs), topical therapies, or watchful waiting. 7 The objective of our

*Address for correspondence and reprint requests: Margaret C. Fang MD, Associate Professor of Medicine, Division of Hospital Medicine, The University of California, San Francisco, 533 Parnassus Ave., Box 0131, San Francisco, CA 94143; Telephone: 415-502-7100; Fax: 415-5142094; E-mail: mfang@medicine.ucsf.edu

Additional Supporting Information may be found in the online version of this article.

Received: August 31, 2015; Revised: December 23, 2015; Accepted: December 31, 2015

2016 Society of Hospital Medicine DOI 10.1002/jhm.2553

Published online in Wiley Online Library (Wileyonlinelibrary.com). study was to describe the initial management of SVTE in a community-based population and examine subsequent rates of diagnosed DVT or PE in the following year.

\section{MATERIALS AND METHODS}

This was a retrospective, observational study seeking to describe the initial treatment for patients diagnosed with isolated SVTE.

\section{Cohort Assembly}

Data for this study were obtained from the Cardiovascular Research Network Venous Thromboembolism cohort study. The source population was based in Kaiser Permanente Northern California (KPNC), a large, integrated healthcare delivery system currently providing comprehensive care for $>3.84$ million members, and comprised of all adults aged 21 years or older with continuous enrollment in the KPNC health plan for $\geq 1$ year and with a primary or secondary International Classification of Diseases, 9th RevisionClinical Modification (ICD-9-CM) diagnosis code of venous thrombosis $(415.1 \mathrm{x}, 451.1 \mathrm{x}, 451.2,451.81$, $453.4 \mathrm{x}, 453.5 \mathrm{x}, 451.83,451.84,451.89,453.72$, $453.73,453.74,453.75,453.76,453.77,453.82$, $453.83,453.84,453.85,453.86,453.87,451,451.9$, 452, 453, 453.0, 453.1, 453.2, 453.3, 453.79, 453.8, 453.89, 453.9) between January 1, 2004 and December 31,2010 . Of the 31,967 individuals meeting these criteria, 930 patients were selected by a random number generator for manual chart abstraction and review. Trained physician reviewers reviewed available emergency department, admission and discharge notes, outpatient clinic notes, and relevant radiology 
TABLE 1. Clinical Characteristics of 329 Patients With Superficial Thrombophlebitis

\begin{tabular}{lc}
\hline Clinical Characteristic & Value, $\mathrm{n}=329$ \\
\hline Age, $y$, mean (standard deviation) & $59.4(15.8)$ \\
Female, $\mathrm{n}(\%)$ & $199(60.5)$ \\
Race, $\mathrm{n}(\%)$ & \\
White & $236(71.7)$ \\
Black & $23(7.0)$ \\
Asian/Pacific Islander & $22(6.7)$ \\
Unknown & $48(14.6)$ \\
Location of thrombophlebitis, $n$ (\%) & \\
Lower extremity & $200(60.8)$ \\
Upper extremity & $108(32.8)$ \\
Other/unknown & $21(6.3)$ \\
Clinical risk factors, $n$ (\%) & \\
Varicose veins & $85(25.8)$ \\
History of recent peripheral intravenous catheters & $71(21.6)$ \\
History of recent local trauma & $22(6.7)$ \\
History of thrombosis & $12(3.7)$ \\
History of stroke & $7(2.1)$ \\
Sepsis/acute infection & $18(5.5)$ \\
Heart failure & $7(2.1)$ \\
Chronic lung disease & $24(7.3)$ \\
Malignant neoplasm & $29(8.8)$ \\
Hospitalization or surgery within 30 days & $48(14.6)$ \\
Hormone therapy & $12(3.6)$ \\
Pregnantpostpartum & $3(0.9)$ \\
Current smoker & $13(4.0)$ \\
Body mass index available & $184(55.9)$ \\
<25 & $48(14.6)$ \\
$>25-30$ & $64(19.5)$ \\
$>30$ & $72(21.9)$ \\
\hline
\end{tabular}

reports to determine whether or not the encounter represented a DVT, a SVTE, or other event.

Episodes were considered isolated SVTE if there was no evidence of a DVT or PE, and if there was medical chart documentation of either a diagnosis of SVTE, ultrasound evidence of a superficial vein clot, or a clinical description of SVTE as determined by the reviewing physician. All SVTE episodes in the study underwent a confirmatory review by second physician reviewer to confirm the diagnosis of SVTE.

\section{Predictors and Outcomes}

The primary outcome was documentation in the medical chart of a treatment recommendation for an antithrombotic agent, specifically, antiplatelet agents (aspirin, clopidogrel, ticlopidine), NSAIDs, and anticoagulants (low-molecular-weight heparin, fondaparinux, or warfarin). The secondary outcome was a subsequent diagnosis of VTE, which we defined as a subsequent encounter with an ICD-9-CM code for DVT or PE within 12 months after the initial episode, accompanied by a prescription for an anticoagulant within 7 days.

Data on patient age, sex, self-reported race/ethnicity, and treatment setting (inpatient, emergency department, or outpatient) were obtained from health plan databases. Clinical risk factors for SVTE and the clinical presentation and treatment were obtained from physician chart review. Assessed risk factors
TABLE 2. Initial Management of 329 Patients

Diagnosed With Superficial Thrombophlebitis

\begin{tabular}{lllccr}
\hline & \multicolumn{4}{c}{ Initial Management, \% (No.) } & \\
\cline { 2 - 5 } VTE Risk* & NSAIDs & LMWH & Warfarin & $\begin{array}{c}\text { No Documented } \\
\text { Antithrombotic Therapy }\end{array}$ & Total \\
\hline Low & $52 \%(128)$ & $1 \%(3)$ & $2 \%(5)$ & $45 \%(112)$ & 248 \\
High & $25 \%(20)$ & $4 \%(3)$ & $4 \%(3)$ & $68 \%(55)$ & 81 \\
Total & $45 \%(148)$ & $2 \%(6)$ & $2 \%(8)$ & $51 \%(167)$ & 329 \\
\hline
\end{tabular}

NOTE: Abbreviations: LMWH, low-molecular-weight heparin; NSAIDs, non-steroidal anti-inflammatory drugs; VTE, venous thromboembolism. *High risk was defined as diagnosis of active malignancy, hospitalization within 30 days, or known hypercoagulable state. Low risk was defined as absence of these risk factors.

included clinical conditions that have been associated with mildly increased SVTE risk (history of tobacco smoking, high body mass index), strongly increased risk (surgery or hospitalization within 30 days, active malignancy, hormonal therapy/pregnant or postpartum), provoking events (local trauma, central or peripheral intravenous catheter placement), and medical conditions that raise the risk for DVT (such as prior history of thrombosis or ischemic stroke). ${ }^{8,9}$ Data were abstracted by a single author (B.T.S.) using a standardized abstraction form. The study was approved by the institutional review boards of the collaborating institutions and informed consent was waived due to the nature of the study.

\section{Statistical Methods}

Analyses were conducted using SAS statistical software version 9.3 (SAS Institute Inc., Cary, NC), with a 2 -sided $P<0.05$ considered significant. We used $\chi^{2}$ tests and Student $t$ tests for categorical and continuous variables, respectively, to test the bivariate association of risk factors with receipt of antithrombotic therapy after SVTE. Multivariable models were not developed due to the limited sample size.

\section{RESULTS}

Out of 930 patients with a diagnosis code for venous thrombosis and who underwent chart review, we identified 329 individuals who were considered by reviewers to have isolated SVTE events. Most SVTEs were of the lower extremity $(60.8 \%)$ and diagnosed in an outpatient or emergency department setting $(91.8 \%)$. Risk factors for SVTE were common, including documented varicose veins, recent peripheral venous catheterization or injection, or antecedent hospitalization (Table 1).

Initial treatment strategies for the 329 patients are presented in Table 2. Few patients with SVTE received anticoagulants for initial treatment, although patients with lower extremity SVTE were more likely to receive antithrombotic therapy compared to patients with SVTE of other locations $(P<0.001)$. None of the identified risk factors for thrombosis were statistically significantly associated with a greater 
likelihood of receiving anticoagulants $(P>0.05$ for all).

In the 12 months after SVTE, $19(5.8 \%)$ patients had a diagnosis encounter for VTE associated with a prescription for either warfarin or parenteral anticoagulant. Of the 200 patients in our study with lower extremity SVTE, $15(7.5 \%)$ had a subsequent VTE diagnosis associated with anticoagulation prescription in the following year.

\section{DISCUSSION}

Clinically significant VTE within a year after SVTE diagnosis was uncommon in our study despite infrequent use of antithrombotic therapy. Although recommendations for the initial treatment of SVTE have evolved in more recent years to support the use of fondaparinux in selected patients, there are significant costs and inconveniences associated with anticoagulation therapy and debate among physicians about the preferred treatment. ${ }^{7}$ The low rate of anticoagulant use in our study may be related to the years studied (before guidelines supported fondaparinux), as well as being largely comprised of outpatients, and also because we included types of SVTE that are unlikely to progress to DVT, such as small vein phlebitis or upper extremity SVTE. ${ }^{4,10}$

Limitations of our analysis include the heterogeneous types of SVTE included in our study and our reliance on available chart documentation to ascertain SVTE diagnosis, risk factors, and treatment. Because of the observational nature of our study, SVTE in the hospital setting may have been less well documented in medical records, leading to a sample of mostly outpatients. Hence, our observed subsequent VTE rate may not be generalizable to a more inclusive population. Finally, the low rate of anticoagulant treatment and VTE diagnoses limited our ability to conduct multivariable modeling.
In conclusion, clinically significant VTE within a year after SVTE was uncommon in our study despite infrequent use of antithrombotic therapy. Although our data are observational, they suggest that not all patients may require anticoagulation for the management of SVTE, and that further investigation into defining which populations would most benefit from treatment with fondaparinux or other agents is warranted.

Disclosures: This study was funded by the National Heart, Lung, and Blood Institute of the National Institutes of Health (grants R01HL103820 and U19HL91179). The sponsor was not involved in the design and conduct of the study; collection, management, analysis, and interpretation of the data; or preparation, review, or approval of the manuscript. Dr. Go received research grant funding from CSL Behring. None of the other authors have financial conflicts of interest.

\section{References}

1. Di Nisio M, Wichers IM, Middeldorp S. Treatment for superficial thrombophlebitis of the leg. Cochrane Database Syst Rev. 2013;4: CD004982.

2. Decousus H, Quere I, Presles E, et al. Superficial venous thrombosis and venous thromboembolism: a large, prospective epidemiologic study. Ann Intern Med. 2010;152:218-224.

3. Cannegieter SC, Horvath-Puho E, Schmidt M, et al. Risk of venous and arterial thrombotic events in patients diagnosed with superficial vein thrombosis: a nationwide cohort study. Blood. 2015;125:229-235.

4. Decousus H, Prandoni P, Mismetti P, et al. Fondaparinux for the treatment of superficial-vein thrombosis in the legs. N Engl J Med. 2010;363:1222-1232.

5. Blondon M, Righini M, Bounameaux H, Veenstra DL. Fondaparinux for isolated superficial vein thrombosis of the legs: a cost-effectiveness analysis. Chest. 2012;141:321-329.

6. Kearon C, Akl EA, Comerota AJ, et al. Antithrombotic therapy for VTE disease: Antithrombotic Therapy and Prevention of Thrombosis, 9th ed: American College of Chest Physicians Evidence-Based Clinical Practice Guidelines. Chest. 2012;141(2 suppl):e419S-e494S.

7. Lane TR, Sritharan K, Herbert JR, Franklin IJ, Davies AH. The disparate management of superficial venous thrombosis in primary and secondary care. Phlebology. 2015;30:172-179.

8. Roach RE, Lijfering WM, van Hylckama Vlieg A, Helmerhorst FM, Rosendaal FR, Cannegieter SC. The risk of venous thrombosis in individuals with a history of superficial vein thrombosis and acquired venous thrombotic risk factors. Blood. 2013;122:4264-4269.

9. Cosmi B, Filippini M, Campana F, et al. Risk factors for recurrent events in subjects with superficial vein thrombosis in the randomized clinical trial SteFlux (Superficial Thromboembolism Fluxum). Thromb Res. 2014;133:196-202.

10. Galanaud JP, Bosson JL, Genty C, et al. Superficial vein thrombosis and recurrent venous thromboembolism: a pooled analysis of two observational studies. J Thromb Haemost. 2012;10:1004-1011. 